\title{
INOCULAÇÃO DE LATÃO A PARTIR DA ADIÇÃO DE FÓSFORO E ZIRCÔNIO*
}

\author{
Thiago Pires Nagasima ${ }^{1}$ \\ Marcelo Alexandre Tirelli ${ }^{2}$ \\ Marcelo de Aquino Martorano ${ }^{3}$ \\ Juan Marcelo Rojas Arango 4 \\ Angelo Fernando Padilha ${ }^{3}$
}

\section{Resumo}

O refino de grão da estrutura bruta de solidificação da liga Cu-30\%Zn (latão 70/30) a partir de adições de $\mathrm{P}$ e $\mathrm{Zr}$ ao banho líquido foi investigado utilizando-se microscopia óptica, microscopia eletrônica e microanálises. Foram conduzidas medidas de tamanho médio de grão pelos métodos planimétrico e do intercepto linear e foram realizadas microanálises por espectroscopia de dispersão de energia (EDS) e de comprimento de onda (WDS). Nas amostras obtidas observa-se que, quando são adicionados $\mathrm{P}$ e $\mathrm{Zr}$, sempre ocorre a diminuição do tamanho médio de grão em relação àquele das amostras onde apenas $\mathrm{P}$ é adicionado. $\mathrm{O}$ menor tamanho médio $(\approx 200 \mu \mathrm{m})$ ocorre para um teor de $0,16 \%$ de $\mathrm{Zr}$. O refino de grão coincide com a formação de precipitados geralmente facetados, contendo $\mathrm{Zr}$ e $\mathrm{P}$ em uma proporção equivalente à da fase ZrP. Sugere-se que as adições causaram a formação de partículas sólidas de ZrP, sobre as quais ocorreu a nucleação heterogênea de grãos da fase $\mathrm{Cu}-\alpha$, resultando no refino de grão.

Palavras-chave: refino de grão; cobre; latão; nucleação heterogênea.

\section{INOCULATION OF BRASS BY PHOSPORUS AND ZIRCONIUM ADDITION Abstract}

Grain refinement of the as-cast macrostructure of a Cu-30\%Zn alloy (70/30 brass) by the addition of $\mathrm{P}$ and $\mathrm{Zr}$ to the melt was investigated using optical microscopy, electronic microscopy, and microanalyses. Measurements of average grain size were carried out by the planimetric and linear intercept methods, and microanalyses were conducted by energy (EDS) and wavelength (WDS) dispersive spectroscopy. In the resulting ingots, the average grain size always decreases when $\mathrm{P}$ and $\mathrm{Zr}$ are added in comparison to the grain size of samples with only $\mathrm{P}$ addition. The smallest average grain size $(\approx 200 \mu \mathrm{m})$ is observed for an addition of $0,16 \% \mathrm{Zr}$. The grain refinement coincides with the formation of faceted precipitates with $\mathrm{Zr}$ and $\mathrm{P}$ concentrations corresponding to those of the ZrP phase. Solid particles of ZrP are believed to form when $\mathrm{P}$ and $\mathrm{Zr}$ are added and heterogeneous nucleation of the $\mathrm{Cu}-\alpha$ phase probably occurs on these particles, causing grain refinement.

Keywords: grain refinement; copper; brass; heterogeneous nucleation.

1 Engenheiro Metalurgista, Bolsista de Mestrado CAPES, Departamento de Engenharia Metalúrgica e de Materiais, Escola Politécnica da Universidade de São Paulo, Av. Prof. Mello Moraes, 2463.

2 Doutor em Engenharia de Materiais, Bolsista CAPES de Pós-Doutorado, Departamento de Engenharia Metalúrgica e de Materiais, Escola Politécnica da Universidade de São Paulo, Av. Prof. Mello Moraes, 2463.

3 Professor do Departamento de Engenharia Metalúrgica e de Materiais, Escola Politécnica da Universidade de São Paulo, Av. Prof. Mello Moraes, 2463, São Paulo, SP, Brasil, CEP 05508-900.

4 Professor do Departamento de Engenharia Metalúrgica e de Materiais, Universidade de Antioquia, Calle 67 No. 53 - 108, Medellín, Colômbia. 


\section{INTRODUÇÃO}

A relativa facilidade para fundição de cobre e suas ligas aliada à combinação de propriedades como a resistência mecânica, a resistência à corrosão e a alta condutividade térmica e elétrica permite a utilização desses materiais em aplicações hidráulicas, artigos decorativos, componentes eletrônicos, buchas, engrenagens e rolamentos, dispositivos expostos a ambientes marítimos e equipamentos nucleares $[1,2]$. O refino de grão é muitas vezes necessário para atender às necessidades de melhores propriedades mecânicas e de fundição. Em ligas de $\mathrm{Cu}$, o refinamento de grãos aumenta o limite de resistência e de escoamento, aumenta a fluidez para a fundição e diminui a ocorrência de trincas a quente [3,4]. Este refino é obtido pelo processo de inoculação, que consiste em realizar adições ao banho metálico para aumentar o número de núcleos de grãos que se formam no início da solidificação, reduzindo o tamanho de grão médio final. Os poucos estudos sobre inoculação em ligas de $\mathrm{Cu}$ são em maioria de caráter tecnológico, muitas vezes realizados a partir de adições simultâneas de elementos ao banho, dificultando o entendimento dos mecanismos que causam a inoculação. No caso das ligas de Al, estes mecanismos já estão bem estabelecidos [5-8]: uma quantidade significativa de partículas sólidas conhecidas, que funcionam como potentes sítios (substratos) para a nucleação heterogênea, são introduzidas com as adições. No caso das ligas de $\mathrm{Cu}$, há alguns estudos tecnológicos, porém poucos têm como objetivo principal entender os mecanismos de inoculação pertinentes. Nos trabalhos de Reif e Weber [9], Romankiewicz et al. [10] e Fasoyinu et al. [11], foi estudado o refino de grão de latões, porém os mecanismos causadores do refino não foram investigados. Bustos et al. [12] apresentou um estudo onde propõe que o refino de grão de latões a partir de adições de $\mathrm{Zr}$ é causado por nucleação heterogênea sobre partículas de $\mathrm{ZrP}$, porém este mecanismo não foi investigado com profundidade. Arango [13] mostrou diversas evidências de que o refino de grão de bronzes ao $\mathrm{Sn}$ a partir de adições de $\mathrm{Zr}$ é causado por nucleação heterogênea sobre partículas de $\mathrm{ZrC}$. O presente trabalho tem como objetivo estudar o refino de grão do latão Cu-30\%Zn (latão 70/30) a partir de adições de $\mathrm{Zr}$ ao banho líquido. As amostras obtidas após o tratamento de inoculação e solidificação foram caracterizadas por meio de suas macroestruturas de grãos e microestruturas observadas em microscópio óptico e eletrônico de varredura além de microanálises por microssonda eletrônica (EDS).

\section{MATERIAIS E MÉTODOS}

Uma liga mãe de composição nominal Cu-30\%Zn foi produzida a partir da fusão em forno de indução de uma carga inicial de Cu denominado "oxygen free" ( $\geq$ $99.95 \% \mathrm{Cu}$ ) e posterior adição de uma carga de $\mathrm{Zn} \mathrm{SHG} \mathrm{(} \geq 99.99 \%)$. Durante todo o período de fusão, injetou-se Ar de pureza comercial a uma vazão de $10 \mathrm{l} / \mathrm{min}$ como proteção gasosa. Após homogeneização, o banho foi vazado a uma temperatura de aproximadamente $1067^{\circ} \mathrm{C}$, resultando em diversos lingotes. Em cada ensaio, alguns destes pequenos lingotes eram posteriormente refundidos em um forno a resistência do tipo mufla, no interior de um cadinho de grafita com tampa de grafita e cobertura de grafite em pó de alta pureza $(99,9 \% \mathrm{C})$. O banho líquido era então desoxidado com a adição de $0,025 \% \mathrm{P}$ a partir de uma liga $\mathrm{Cu}-15 \% \mathrm{P}$. Uma parte do banho era então vazada em um sistema de solidificação (Figura 1) e, posteriormente, adicionava-se $\mathrm{Zr}$ na forma de $\mathrm{Cu}-50 \% \mathrm{Zr}$ até o teor de $\mathrm{Zr}$ desejado para então realizar o vazamento do restante do banho. Desta forma, em cada ensaio sempre 
foram obtidas duas amostras aproximadamente nas mesmas condições térmicas, porém uma sem e outra com a adição de $\mathrm{Zr}$. Todos os vazamentos foram realizados após o termopar do forno indicar $1060^{\circ} \mathrm{C}$. O sistema de solidificação (Figura 1) foi constituído por um copo descartável cilíndrico de areia-shell contendo em seu centro dois termopares do tipo $\mathrm{K}$ (Cromel-Alumel) com isolação mineral e protegido por uma bainha de aço inox 310 , de diâmetro igual a $1,5 \mathrm{~mm}$. Os termopares foram protegidos com duas camadas de tinta: uma isolante (Firit 5) e a outra condutora à base de grafite (Dycote 11), produzidas pela Foseco. O sistema de aquisição de dados é composto por um computador pessoal com o software LabView (National Instruments), uma placa contendo um conversor analógico-digital com resolução de 16 bits (National Instruments) e um condicionador de sinais. Os dois termopares foram conectados ao sistema de aquisição por meio de fios de compensação blindados com trança metálica. O sistema registrou os sinais de tensão emitidos pelo termopar, posteriormente convertidos para temperatura, a uma taxa de 10 medidas por segundo.

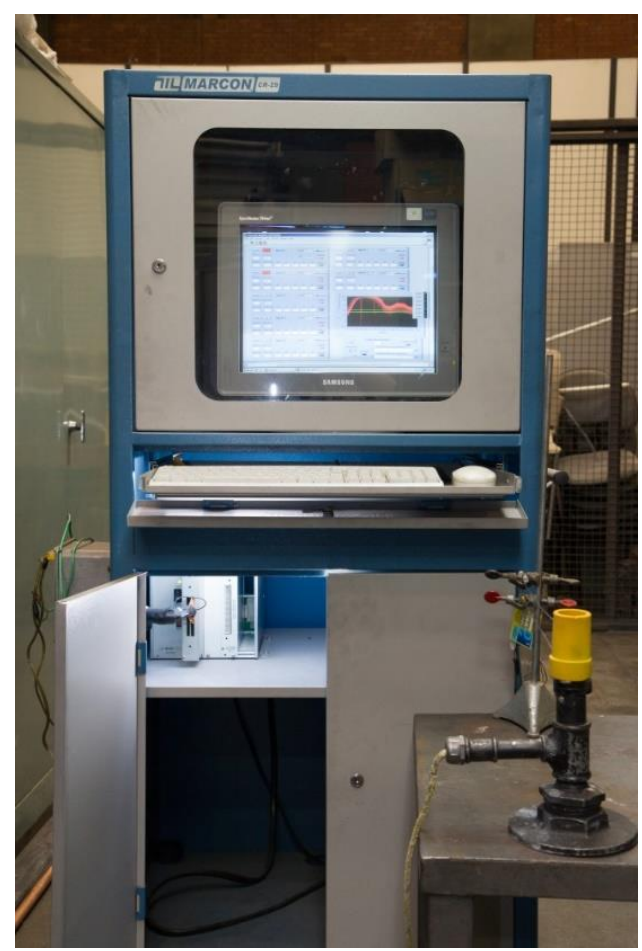

(a)

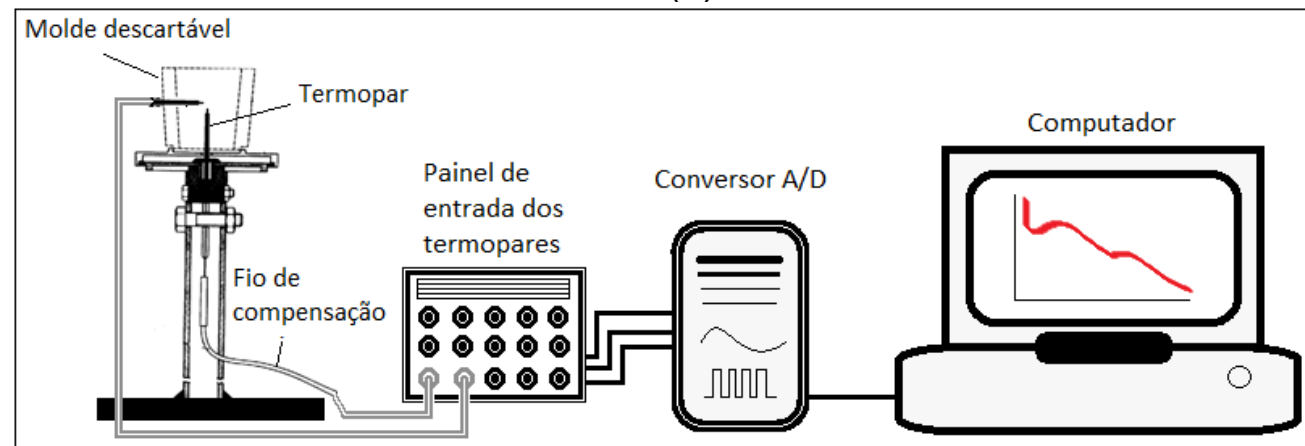

(b)

Figura 1. Sistema de solidificação: a) Sistema com copo de areia shell posicionado sobre uma base; b) Desenho esquemático do sistema de solidificação e o sistema de aquisição de dados. 
Os lingotes cilíndricos obtidos nos experimentos foram cortados longitudinalmente. Uma das metades foi preparada por técnicas metalográficas convencionais e posteriormente atacada (30\% de $\mathrm{HNO}_{3}$ em água) para revelar a macroestrutura de grãos. A medida do tamanho médio de grão foi conduzida sobre esta macroestrutura a partir dos métodos do intercepto circular e planimétrico, descritos no padrão ASTM E112-96 [14]. As amostras para observação em microscópio óptico e eletrônico foram lixadas e polidas utilizando técnicas metalográficas tradicionais, sendo algumas delas atacadas com reagente químico (5g de $\mathrm{FeCl}_{3}, 100 \mathrm{~mL}$ de etanol e $5 \mathrm{~mL}$ de $\mathrm{HCl}$ ).

\section{RESULTADOS E DISCUSSÃO}

\subsection{Refino de Grão do Latão 70/30}

A Figura 2 apresenta as macroestruturas de grãos das amostras com e sem adição de $\mathrm{Zr}$ para teores crescentes de $\mathrm{Zr}$. Pode-se verificar que a adição de $\mathrm{Zr}$ na faixa de 0,02 a 0,4\% sempre diminuiu o tamanho de grão do latão 70/30 em comparação a amostra sem adição. A Figura 3 apresenta as medidas de tamanho de grão médio em função do teor nominal de Zr. O tamanho de grão da amostra inoculada diminui com o aumento do teor de $\mathrm{Zr}$, atingindo um valor mínimo $200 \mu \mathrm{m}$ para $0,16 \% Z r$ e aumentando discretamente após este teor. Este comportamento é semelhante ao encontrado por Arango [16] na inoculação da liga Cu-8\%Sn, onde o tamanho de grão teve queda substancial até a adição de $0,08 \%$ (massa) de $\mathrm{Zr}$ e passou a aumentar para teores maiores que 0,24\%. Nota-se também que há uma boa correlação entre as medidas de tamanho de intercepto pelos dois métodos utilizados, o planimétrico e do intercepto circular, aumentando a confiabilidade das medidas.

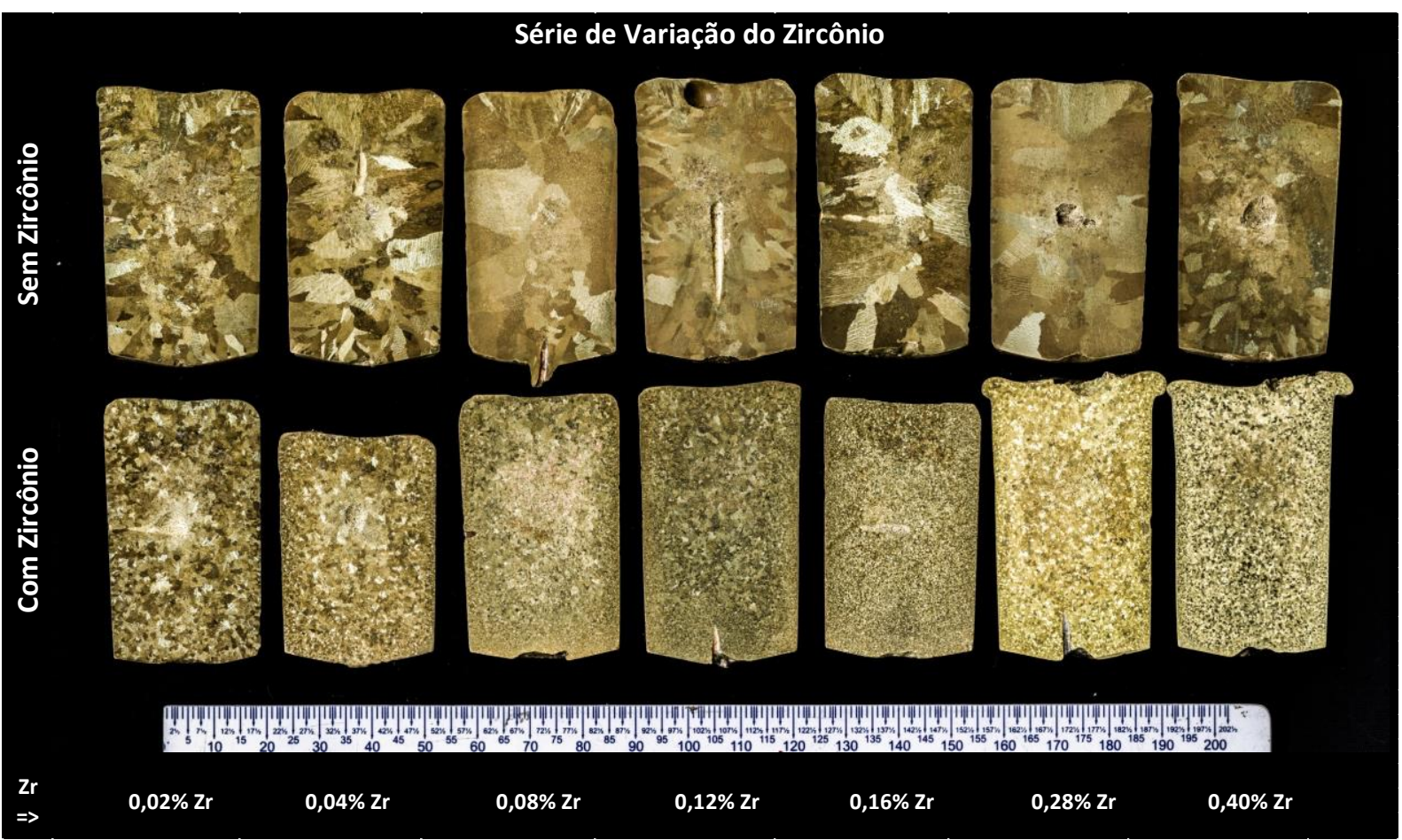

Figura 2. Macroestruturas da seção transversal da série dos experimentos com adição crescente de $\mathrm{Zr}$. Acima, amostras sem adição de $\mathrm{Zr}$ e abaixo com as adições. 


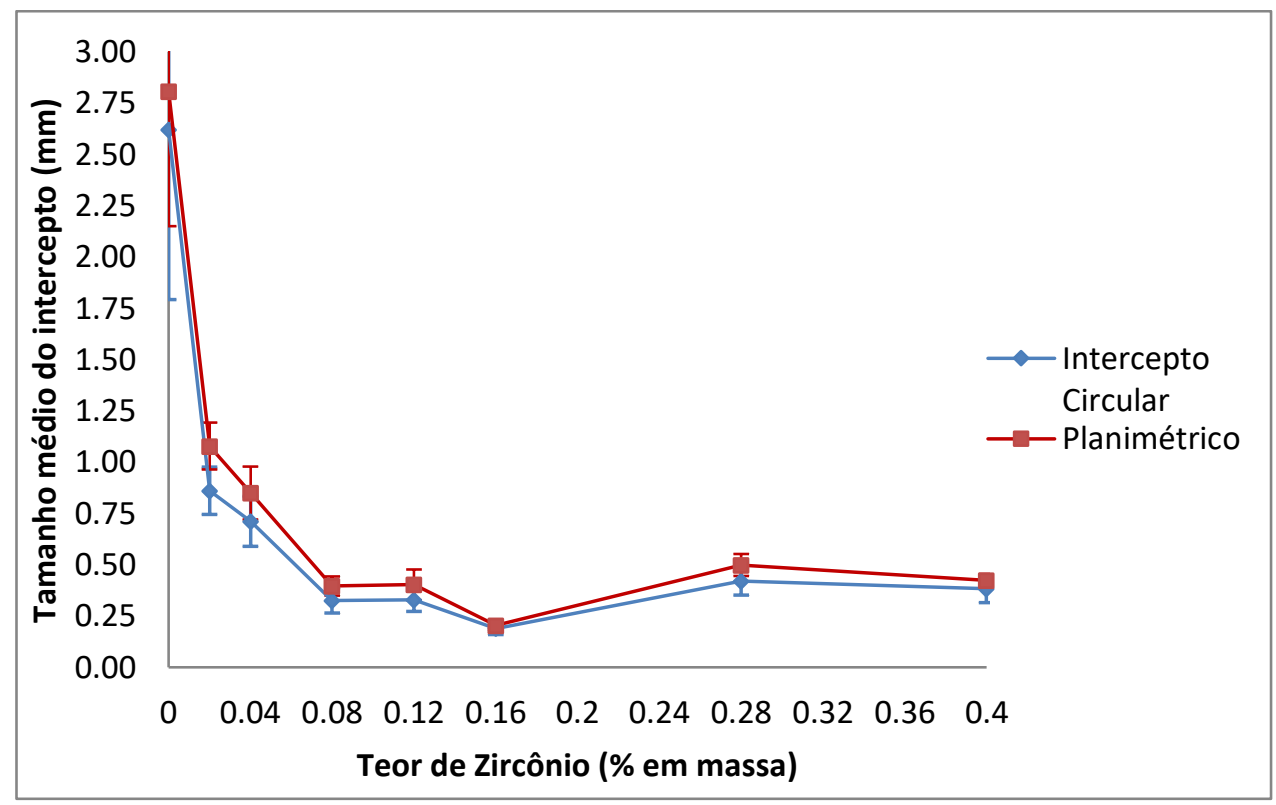

Figura 3. Curva de tamanho de grão médio para diferentes adições nominais de Zr.

Como descrito no item Materiais e Métodos, em cada ensaio sempre foi obtida uma amostra sem a adição de $\mathrm{Zr}$ para comparação com a amostra onde o $\mathrm{Zr}$ foi adicionado. Todas as amostras sem $\mathrm{Zr}$ foram obtidas nas mesmas condições experimentais e, portanto, as variações em sua macroestrutura mostram o efeito das pequenas alterações inerentes ao procedimento experimental adotado, como por exemplo pequenas variações na temperatura de vazamento [9]. Desta forma, é possível verificar visualmente que a redução de tamanho de grão causada pela adição de $\mathrm{Zr}$ não pode ser resultado destas pequenas alterações inevitáveis do procedimento experimental, mas sim de algum efeito adicional, que foi atribuído à inoculação.

\subsection{Análise Microestrutural}

A microestrutura de uma amostra obtida sem adição de $\mathrm{Zr}$ está mostrada na Figura 4 em uma imagem de microscopia óptica, onde se observa a presença de uma típica estrutura dendrítica revelada pelo ataque químico. Em maiores aumentos, algumas partículas podem ser observadas, como mostra a Figura 5, em uma imagem de microscopia óptica sem (Figura 5a) e com (Figura 5b) ataque químico. A microanálise por espectroscopia de dispersão de energia (EDS) ilustrada na Figura 6 mostra que este precipitado contém basicamente $\mathrm{Cu}, \mathrm{P}$ e $\mathrm{O}$ e pode ser a fase $2 \mathrm{Cu}_{2} . \mathrm{P}_{2} \mathrm{O}_{5}$ [1], geralmente resultante da desoxidação do banho líquido com $\mathrm{P}$. Algumas destas partículas também foram encontradas na amostra com adição de $0,02 \% \mathrm{Zr}$, mas não nas amostras com maiores teores de Zr. Todas as amostras com adição de Zr, por sua vez, apresentaram precipitados geralmente facetados em formatos hexagonais e de bastonetes, como mostra a Figura 7. Esta morfologia é similar àquela observada por Arango [13] após inoculação de bronzes ao estanho (Cu-8\%Sn) com adição de Zr. 


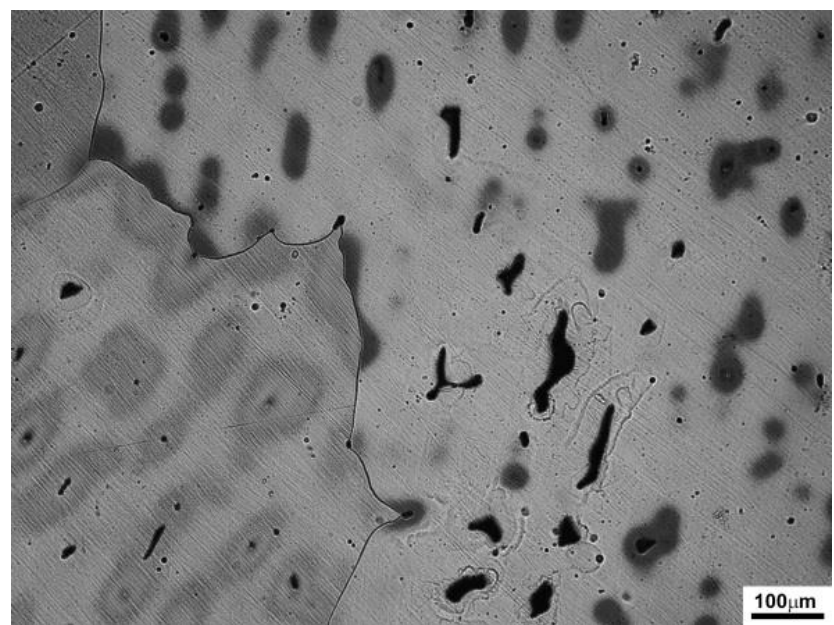

Figura 4. Microestrutura de uma amostra de latão 70/30 sem adição de $Z$ r, revelada por ataque químico e observada em microscópio óptico.
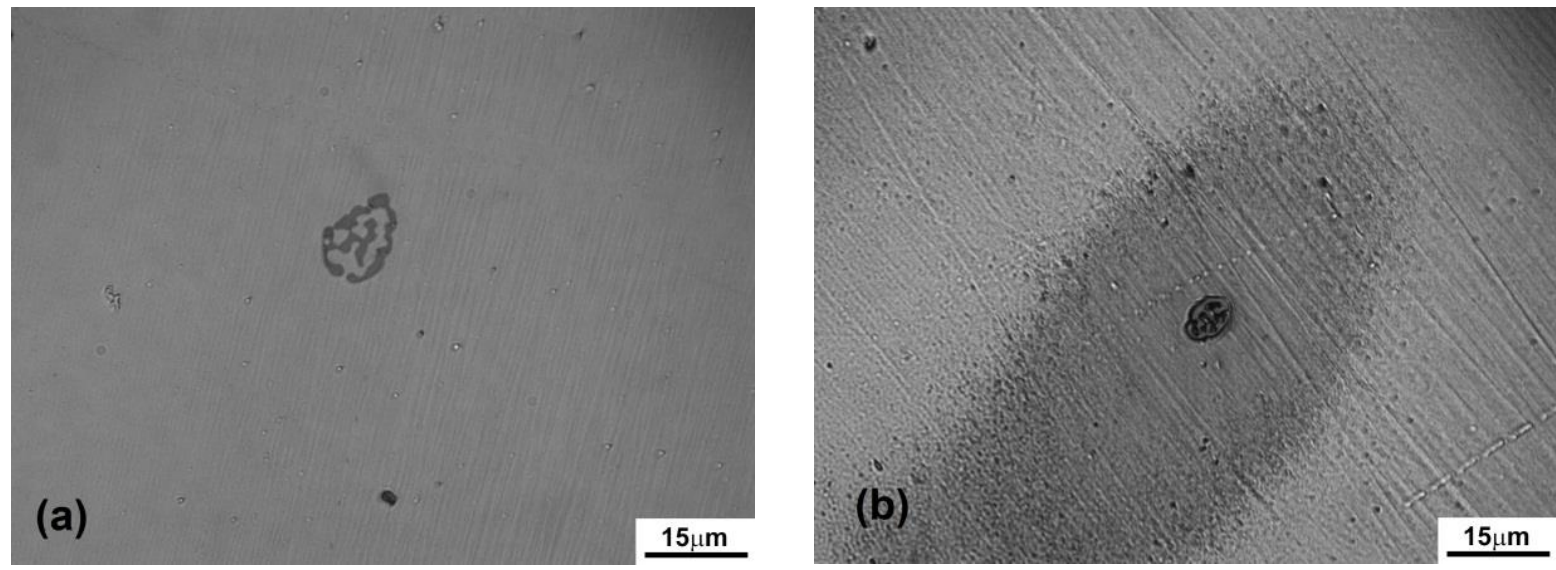

Figura 5. Microestrutura observada em microscópio óptico de uma amostra sem adição de Zr: (a) sem e (b) com ataque químico.
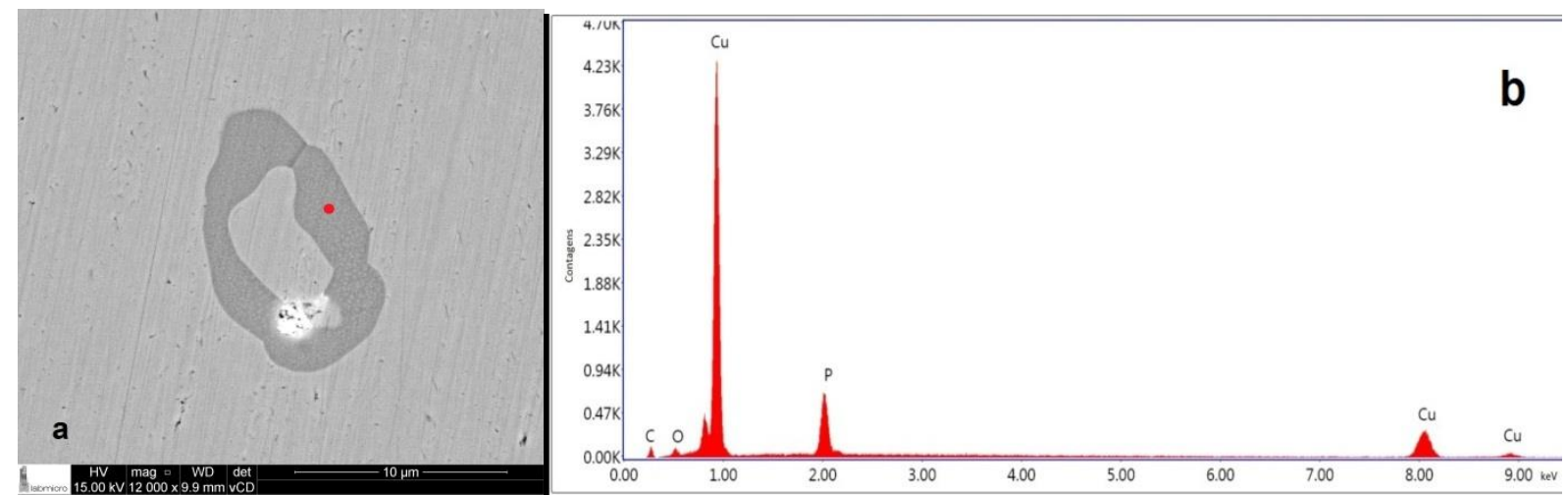

Figura 6. Análise de uma partícula típica encontrada nas amostras sem adição de Zr e com 0,02\%Zr: a) imagem de contraste de elétrons retroespalhados em MEV-FEG; b) espectro de EDS do ponto indicado em (a). 


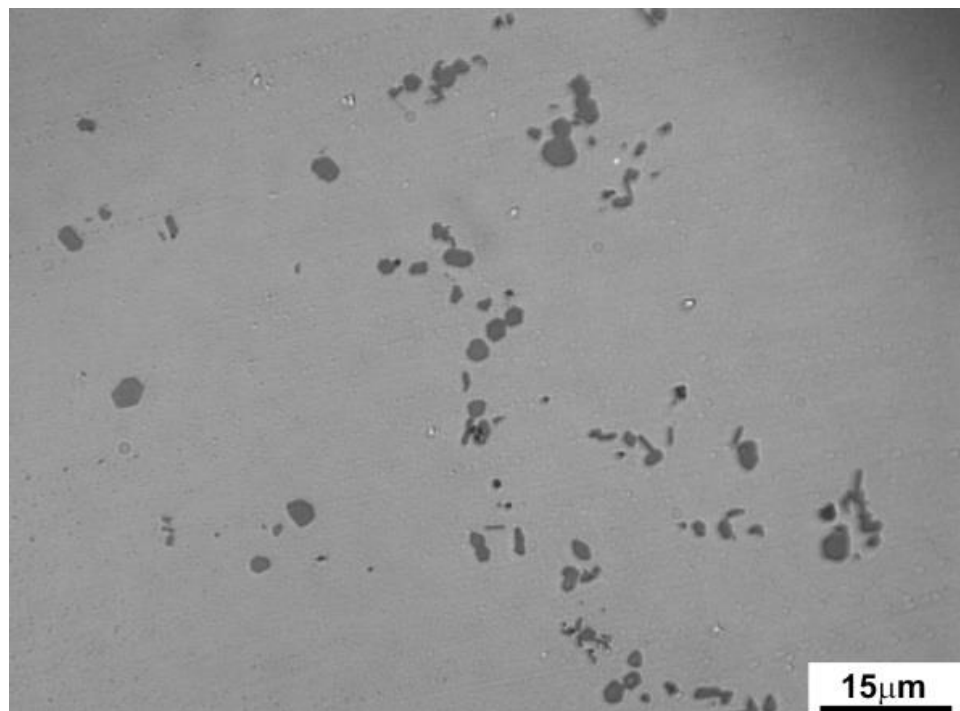

Figura 7. Micrografia de microscópio óptico de uma amostra com adição de $0,16 \%$ Zr sem ataque químico.

Uma microanálise por EDS típica sobre estes precipitados está apresentada na Figura 8(b) ao lado da micrografia obtida em MEV-FEG (Figura 8(a)). Na Figura 8(b), o sistema automático de identificação de elementos indicou a presença principalmente de picos de $\mathrm{P}, \mathrm{Zr}$ e $\mathrm{Cu}$. O pico de $\mathrm{C}$ é quase imperceptível e deve ser analisado com cuidado, pois a técnica de EDS é limitada na faixa de energia onde se encontra este pico. Além disso, os picos do $\mathrm{P} \mathrm{Ka}(2,013 \mathrm{keV})$ e do $\mathrm{Zr} \mathrm{La}$ $(2,042 \mathrm{keV})$ apresentam uma diferença de energia muito reduzida para ser diferenciada na microanálise EDS [17] e, portanto, não é possível concluir quais destes dois elementos está realmente presente.


Figura 8. Análise da amostra com adição de 0,08\%Zr: a) imagem de partículas típicas em MEV-FEG; b) espectro EDS do ponto indicado em (a).

As amostras com teores de $0,28 \%$ e $0,40 \%$ de $\mathrm{Zr}$ apresentaram, além dos precipitados facetados, uma segunda fase alongada e afinada onde a microanálise EDS novamente indicou a presença de $\mathrm{Zr}$ e/ou $\mathrm{P}$ (Figura 9), mas também a existência de Cu e Zn, que podem ser originários da matriz. 

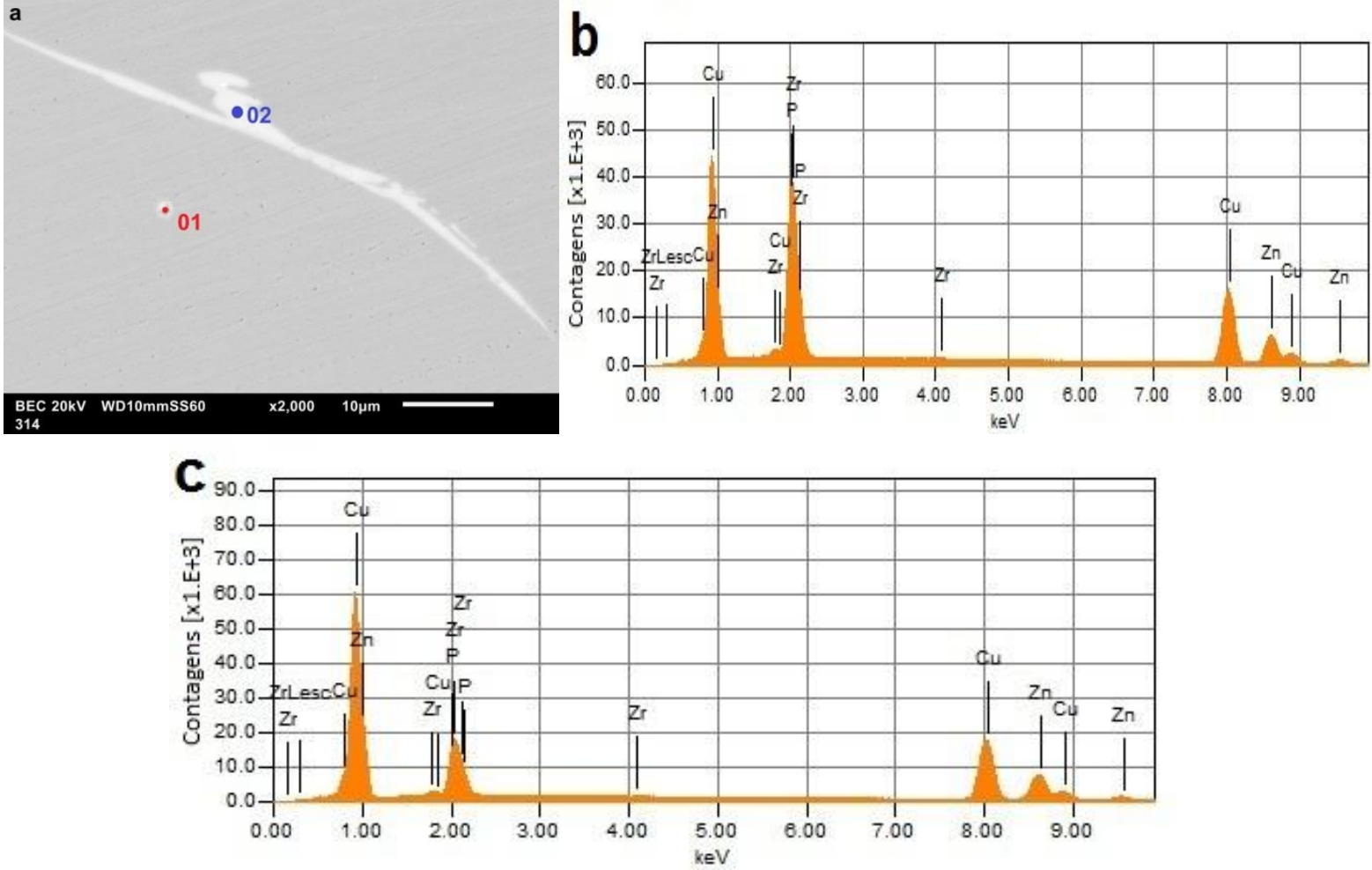

Figura 9. Precipitado na amostra com adição de 0,4\%Zr: a) imagem de elétrons retroespalhados em MEV; b) espectro de dispersão de energia (EDS) no ponto 01 e c) espectro de dispersão de energia (EDS) no ponto 02.

Microanálises por espectroscopia de dispersão de comprimento de onda (WDS) foram conduzidas sobre as partículas facetadas das amostras com adições de $0,08 \%$ e $0,16 \% Z r$, pois esta técnica tem resolução suficiente para diferenciar os picos Zr La e P Ka. Uma calibração cuidadosa dos elementos Zr, P, Cu, Zn e S (os últimos três acusados em algumas das análises EDS) foi conduzida para obtenção da análise quantitativa. $O$ elemento $S$ não foi detectado e as concentrações dos outros elementos para alguns precipitados examinados estão apresentadas na Tabela 2. Com o auxílio de WDS foi possível verificar a presença tanto do $\mathrm{Zr}$ como do $\mathrm{P}$ no precipitado. Nota-se que a soma de todas as concentrações de cada análise totaliza aproximadamente 100\%, indicando consistência. Como mencionado, a presença de $\mathrm{Cu}$ e $\mathrm{Zn}$ pode ser um efeito de interferência da matriz, que é uma solução sólida de Cu-Zn. Verifica-se que em todas as análises, o teor em massa de $\mathrm{Zr}$ é próximo ao triplo do teor de $\mathrm{P}$, correspondendo à fórmula estequiométrica do $\mathrm{ZrP}$, que é uma possível fase resultante da combinação de $\mathrm{Zr}$ e $\mathrm{P}$ [18]. Logo, acredita-se que este precipitado seja a fase $\mathrm{ZrP}$, formada pelas adições de $\mathrm{Cu}$ $15 \% \mathrm{P}$ (desoxidação) e de Cu-50\%Zr, e que a inoculação da solução sólida de Cu-Zn ocorreu sobre estas partículas, causando o refino do tamanho médio de grão final. Esta evidência confirma a hipótese sugerida por Bustos [12]. 
Tabela 2. Teores em \% massa de $\mathrm{Cu}, \mathrm{Zn}, \mathrm{Zr}, \mathrm{P}$ e a soma de todas estas concentrações obtidas por microanálise WDS sobre os precipitados facetados encontrados nas amostras com adição de $0,08 \%$ e $0,16 \% Z r$.

\begin{tabular}{cccccc}
\hline $\begin{array}{c}\text { Adição } \\
\text { de } \mathrm{Zr}\end{array}$ & $\% \mathrm{Cu}$ & $\% \mathrm{Zn}$ & $\% \mathrm{Zr}$ & $\% \mathrm{P}$ & Soma \\
\hline $0.08 \% \mathrm{Zr}$ & 8.840 & 4.174 & 67.112 & 20.734 & 100.860 \\
\hline $0.08 \% \mathrm{Zr}$ & 7.866 & 2.792 & 71.180 & 20.274 & 102.112 \\
\hline $0.08 \% \mathrm{Zr}$ & 11.594 & 5.082 & 69.679 & 20.080 & 106.435 \\
\hline $0.16 \% \mathrm{Zr}$ & 4.291 & 1.749 & 77.485 & 22.365 & 105.891 \\
\hline $0.16 \% Z r$ & 5.133 & 1.925 & 76.953 & 22.590 & 106.602 \\
\hline $0.16 \% Z r$ & 4.614 & 1.776 & 77.756 & 22.715 & 106.862 \\
\hline
\end{tabular}

\section{CONCLUSÕES}

Banhos líquidos de Cu-30\%Zn (latão 70/30) foram desoxidados com Cu-15\%P e posteriormente receberam adições de $\mathrm{Cu}-50 \% \mathrm{Zr}$ em diferentes quantidades, resultando em teores nominais de $\mathrm{Zr}$ que variaram em uma faixa de 0,02\% a 0,4\%. Os banhos foram posteriormente vazados e as macroestruturas das amostras obtidas mostraram que estas adições causam uma diminuição significativa no tamanho médio de grão, quando comparado com amostras com adição de $P$, mas sem adição de $\mathrm{Zr}$. A diminuição do tamanho médio de grão causada pelas adições coincide com o aparecimento de precipitados geralmente facetados contendo $\mathrm{Zr}$ e $\mathrm{P}$ em uma proporção equivalente à fase $\mathrm{ZrP}$. Os resultados indicam que as adições de $\mathrm{P}$ e $\mathrm{Zr}$ ao banho líquido de $\mathrm{Cu}-30 \% \mathrm{Zn}$ formam partículas de $\mathrm{ZrP}$, sobre as quais ocorrem a nucleação heterogênea da solução sólida de $\mathrm{Cu}-\mathrm{Zn}$ (fase $\mathrm{Cu}-\alpha$ ), ou seja, as adições de $\mathrm{P}$ e $\mathrm{Zr}$ causam a inoculação e o refino de grão do latão 70/30.

\section{Agradecimentos}

Os autores agradecem a bolsa de estudos concedida pela CAPES, ao suporte financeiro da FAPESP (processo $\mathrm{n}^{\circ}$ 2013/08723-1), ao CNPq (processo 311206/2014-0), à Termomecânica S.A. e à Paranapanema S.A. pelas ligas fornecidas e ao laboratório de Corrosão e Proteção do IPT (em especial à colaboradora Juliana Pereira Flor) pela análise WDS realizada.

\section{REFERÊNCIAS}

1 ASM International. ASM Specialty Handbook - Copper and Copper Alloys. 1a Edição. Materials Park: ASM International, 2001.

2 Joseph G. Copper: Its trade, manufacture, use and enviromental status. $2^{\mathrm{a}}$ Edição. Materials Park: ASM International, 2001.

3 Couture A, Edwards JO. Grain refinement of sand cast bronzes and its influence on their properties. AFS Transactions. 1973;82:453-61.

4 Sadayappan M, Thomson JP, Elboujdaini M, Ping Gu G, Sahoo M. Grain Refinement of Permanent Mold Cast Copper Base Alloys. US Department of Energy, 2004.

5 Cibula A. Mechanism of grain refinement of sand castings in aluminum alloys. J. Inst. Met. 1949; 76:361-376.

6 Backerud L. Grain refining mechanism in Al-Ti-B Alloys. Jernkontorets Annaler. 1971; 155:421.

7 Spittle JA. Grain Refinement in shape casting of aluminum alloys. International Journal of Cast Metals Research. 2006; 210-222. 
8 Fan Z, Wang Y, Zhang Y, Qin T, Zhou XR, Thompson GE, Pennycook T, Hashimoto T. Grain refinement mechanism in the Al/Ak-Ti-B system. Acta Materialia. 2015; 84:292304.

9 Reif W, Weber G. A new grain refiner for copper-zinc alloys containing 25-42\% Zinc. Metallwissenschaft Technik. 1987; p. 1131.

10 Romankiewicz F, Ellerbrok R, Engler S. Einfluß einer Kornfeinung mitZirconium auf Erstarrungsmorphologie, Speisungsvermögen und Festigkeitseigenschaften von Messing CuZn30 und Siliciummessing CuZn15S14. Giessereiforschung. 1987; V.39:2533.

11 Fasoyinu FA, Sadayappan M, Cousineau D, Zavadil R, Sahoo M. Effects of grain refinement on hot tear resistance and shrinkage characteristics of permanent cast yellow brasses (C85800). AFS Transactions. 1998; 106:327-337.

12 Bustos O, Mannheim R, Cruz L. Estudio de la combinación de los processos de afinamento de grano de colada y filtrado en latones. Rev. Metal. Madrid. 1999; 35:222232.

13 Arango JMR. Refino de grão das ligas do sistema cobre-estanho. Tese apresentada à Escola Politécnica da Universidade de São Paulo para obtenção do título de Doutor em Ciências. 2016.

14 ASTM International. E 112 - 96 (Reapproved 2004): Standard Test Methods for Determining Average Grain Size. 2004.

15 Arango JMR, Martorano MA, Padilha AF. Estudo do refino de grão da estrutura bruta de solidificação da liga Cu-8\%Sn. $70^{\circ}$ Congresso Anual da ABM - Internacional.

16 Arango JMR, Nagasima TP, Martorano MA, Padilha AF. Inoculação de bronzes a partir de adições de Zircônio. 71ำ Congresso Anual da ABM - Internacional

17 Newbury DE. Mistakes encountered during automatic peak identification of minor and trace constituents in electron-excited energy dispersive X-Ray microanalysis. Scanning, 2009;31: p.91-101.

18 Irani KS, Gingerich KA. Structural transformation of zirconium phosphide. J. Phys. Chem. Solids, 1963;24: p.1153-1158. 